doi:10.1016/j.jhin.2005.08.006

Copyright (c) 2005 The Hospital Infection Society Published by Elsevier Ltd.

\title{
Random effect modelling of patient-related risk factors in orthopaedic procedures: results from the Dutch nosocomial infection surveillance network 'PREZIES'
}

\author{
J. Muilwijka, G.H.I.M. Walenkamp ${ }^{b}$, A. Voss ${ }^{c}$, J.C. Wille ${ }^{d}$ and S. van den \\ Hof $^{\text {a, } *}$
}

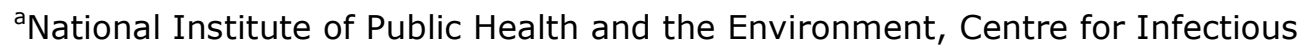
Disease Epidemiology, Bilthoven, The Netherlands

${ }^{b}$ Department of Orthopaedic Surgery, Academic Hospital Maastricht, Maastricht, The Netherlands

${ }^{\mathrm{C}}$ Department of Medical Microbiology, University Medical Centre St Radboud, Nijmegen, The Netherlands

${ }^{\mathrm{d} D u t c h}$ Institute for Healthcare Improvement, Utrecht, The Netherlands

* Corresponding author. Tel.: +31 30274 3685; fax: +31 302744409.

\section{Summary}

In the Dutch surveillance for surgical site infections (SSIs), data from 70277 orthopaedic procedures with 1895 SSIs were collected between 1996 and 2003. The aims of this study were: (1) to analyse the trends in SSIs associated with Gram-positive and Gram-negative bacteria; (2) to estimate patient-related risk factors for deep and superficial SSIs after all orthopaedic procedures, with special attention to primary total hip arthroplasty (THA); and (3) to analyse inherent differences in infection risk between hospitals. A random effect model was used to estimate the odds ratios of patient-related risk factors for developing an SSI, and to describe the distribution of the most widespread bacterial species responsible for SSIs among hospitals. Gram-positive organisms, mainly staphylococci, were the main cause of both deep $(84.0 \%)$ and superficial SSIs $(69.1 \%)$ after orthopaedic procedures. The percentage of SSIs after THA caused by coagulasenegative staphylococci decreased over the surveillance period, while the contribution of Staphylococcus aureus increased. Temporary elevations in the incidence of the most widespread pathogen species were observed within hospitals. Patient-related factors such as the National Nosocomial Infections Surveillance System risk index or age had little effect on the predictive power of the random effect models. This study underlines the usefulness of a random effect model, which adjusts risk estimates for random variation between hospitals, in a multicentre study on risk factors for SSIs.

\section{Introduction}

The development of surgical site infections (SSIs) after orthopaedic procedures is influenced by factors relating to the patient, the type of operation, the environment of the operation room, and the characteristics of the pathogen. ${ }^{1}$

Prevention of SSIs is important, especially in operations involving prostheses. Deep SSI after the implantation of an orthopaedic prosthesis in orthopaedic 
surgery requires a prolonged course of antibiotic treatment and may even lead to the removal and replacement of the prosthesis. ${ }^{2}$ Deep SSIs are generally caused during the operation by contiguous or haematogenous spread. ${ }^{2}$ Superficial infections due to wound healing problems, such as haematoma or persistent wound drainage, may subsequently develop into a deep SSI. ${ }^{3}$ The Dutch Working Party on Infection Prevention (WIP) has published guidelines about the prevention of infection in operating rooms. ${ }^{4}$ Surveillance of SSIs forms an essential part of prevention. $^{5}$

SSIs after orthopaedic operations are predominantly caused by staphylococci, ${ }^{6}$ especially Staphylococcus epidermidis and Staphylococcus aureus, both part of the human skin flora. Among the Gram-negative bacteria, Enterobacteriaceae spp. originating from the intestinal tract and mucous membranes are most prevalent. $^{1}$

A number of publications have recorded outbreaks of hospital infections due to a specific pathogen. ${ }^{7 \text { and } 8}$ Multicentre analysis seldom takes such outbreaks into account, although failure to account for differences between hospitals caused by these outbreaks can result in distorted estimations of relative risks and variances. ${ }^{9}$

The aims of this study were to analyse the trends in SSIs associated with Grampositive and Gram-negative bacteria, and to estimate the odds ratios (OR) of the well-known patient-related risk factors for deep and superficial SSIs in orthopaedic procedures registered within the Dutch national surveillance of nosocomial infections. Special attention was given to primary total hip arthroplasty (THA) as this procedure represents the bulk of all registered orthopaedic procedures.

\section{Methods}

\section{Patient population and data collection}

The surveillance network directed at the prevention of nosocomial infections (PREZIES) in The Netherlands started in $1996 .{ }^{10}$ Hospital participation in PREZIES is voluntary and confidential.

'The surveillance of SSIs includes all patients aged one year upwards. Hospitals are free to choose which type of surgery they want to record. Data collection for each patient occurs according to the PREZIES protocol. ${ }^{11}$ The acquired data entries of the patients and the hospitals are anonymous. Double and illogical data entries were not included in this study.

PREZIES uses the Centers for Disease Control and Prevention's (CDC) definition of SSI, ${ }^{1}$ translated by the WIP. According to the CDC, an SSI must occur within 30 days of the operation if no non-human implant has been left in place, or within one year if an implant has been placed. In the analysis, deep incisional SSIs and organ/space SSIs were evaluated together as deep SSIs. ${ }^{12}$

Over a period of eight years, 73 hospitals have collected data about more than 150 different types of orthopaedic procedures.

\section{Orthopaedic procedures}


Most orthopaedic procedures were elective. Implantation of a prosthesis, a wellknown risk factor for SSI development, ${ }^{1}$ took place in $65 \%$ of the registered orthopaedic procedures. The Dutch Orthopaedic Society has formulated guidelines for the administration of antibiotic prophylaxis with THA and the use of antibioticcontaining bone cement. ${ }^{13}$ Nevertheless, hospitals differed in the use of antibiotic prophylaxis (e.g. number of doses per day). Within PREZIES, only the fact that antibiotic prophylaxis was administered is recorded. In this analysis, THA does not include revision procedures of infected hips.

\section{Micro-organisms}

S. epidermidis, S. haemolyticus, S. simulans, S. lugdunensis and S. saprophyticus were grouped together as coagulase-negative staphylococci (CNS). Trend analyses on incidence of the pathogens were performed on SSIs with only one cultured pathogen and the polymicrobial cultures with only Gram-positive or only Gram-negative bacterium. Information about the type (superficial/deep) of infection was not stated in 31 records, of which 10 had microbiological results available including three with polymicrobial cultures. These 31 data entries were omitted from the analyses of deep vs superficial SSIs. All microbiological culture results were used for analysis of the distribution of pathogens among the hospitals.

\section{Data analysis}

Data were analysed using SAS for Windows, release 8.2 (SAS Institute, Cary, NC, USA). A random effect model (proc NLMIXED in SAS) was used for simultaneously estimating the ORs of the risk factors and the random effects caused by differences between hospitals. The random effect model applied in these analyses is essentially a logistic regression model, with the addition of an extra term in the equation for the random effects associated with differences in infection risk between hospitals. This model is also called a random intercept model. ${ }^{14}$ The estimated variance of the random effects in the model is a measure of the variability between hospitals.

Factors associated with an SSI with $P<0.2$ in an univariate analysis were included in the multivariate model. The final model was made with a manual stepwise backwards elimination. Variables remained in the multivariate model if the likelihood ratio test was significant $(p<0.05)$.

The next step was the calculation of the predicted probability of getting an SSI for each patient, with and without an SSI, using the final model. These probabilities were averaged for both groups separately. Finally, these two means were divided. This ratio was used as a measure of the goodness of fit of the model. A ratio of 1 indicates that the risk factors in the model do not contribute to the prediction of developing an SSI.

A measure for the distribution of pathogens among the hospitals, which is independent of the number of operations performed in a hospital, was found by limiting the analysis with the random effect model to SSIs caused by a single species. In this case, the estimate of the variance of the random hospital effects was taken as a measure for the distribution of this pathogen. If this variance was significant, the species was considered to be clustered.

In total, 70277 orthopaedic procedures, including 26127 THAs, were recorded in PREZIES between 1996 and 2003. At the time of these analyses, the number of data entries for 2003 was less than in preceding years as not all data for 2003 
Journal of Hospital Infection Volume 62, Issue 3 , March 2006, Pages 319-326

had been received. Therefore, the 2003 data were not used in the trend analyses of SSI incidence. With the multivariate analyses, only data entries without missing values for the explored risk factors were used ( $N=64598)$. 
Journal of Hospital Infection Volume 62, Issue 3 , March 2006, Pages 319-326

\section{Results}

Incidence of SSIs

Table I shows the incidence of SSIs after all orthopaedic procedures. A time trend was only observed for superficial SSIs after THA; the yearly incidence was $2.1 \%$ over the period 1996-2000 and 1.3\% over the period 2001-2002 ( $P=0.0002)$. Out of all 1895 SSIs, 1269 cases (67\%) were documented microbiologically. Of these, $301(24 \%)$ had a polymicrobial culture of which 89 contained Grampositive bacteria alone and 37 contained Gram-negative bacteria alone. In both deep and superficial SSIs, $24 \%$ of the cultures were polymicrobial.

\section{Table I.}

Number of surgical site infections (SSIs), mean surgical site infection rate (\%), and minimum and maximum yearly rates (\%) following all orthopaedic procedures and following total hip arthroplasty (THA) alone in the period 19962003

\begin{tabular}{|l|l|l|c|c|c|c|}
\hline \multicolumn{2}{|c|}{} & \multicolumn{2}{|c|}{ All procedures (N=70 277) } & \multicolumn{3}{c|}{ THA (N=26 127) } \\
\hline & Deep & Superficial & $\begin{array}{c}\text { Not } \\
\text { classified }\end{array}$ & Deep & Superficial & $\begin{array}{c}\text { Not } \\
\text { classified }\end{array}$ \\
\hline
\end{tabular}


decrease in both deep and superficial infections between 1996 and 2003, while the proportion of deep infections caused by $S$. aureus seemed to increase (Figure 1 and Figure 2).

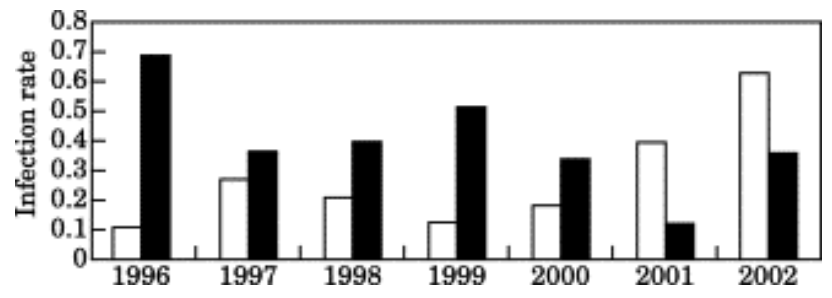

Figure 1. Surgical site infection (SSI) rate caused by Staphylococcus aureus after total hip arthroplasty in the period 1996-2002. Open bars, deep SSIs; solid bars, superficial SSIs.

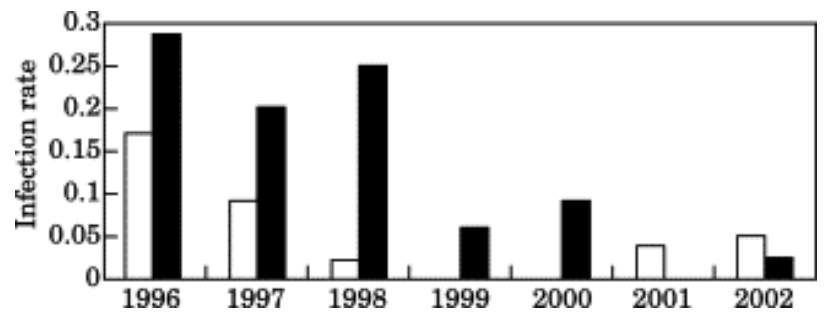

Figure 2. Surgical site infection (SSI) rate caused by coagulase-negative staphylococci after total hip arthroplasty in the period 1996-2002. Open bars, deep SSIs; solid bars, superficial SSIs.

\section{Description of the patient population}

Patient characteristics and results of univariate analysis are shown in Table II. Not all registration forms were complete for all factors. Overall numbers are recorded in Table I. 
Table II.

Number of patients with a surgical site infection (SSI) and rate (\%) per risk factor in univariate analysis following all orthopaedic procedures and following total hip arthroplasty (THA) alone

\begin{tabular}{|c|c|c|c|c|c|c|c|c|}
\hline & \multicolumn{4}{|c|}{ All procedures } & \multicolumn{4}{|c|}{ THA } \\
\hline & \multicolumn{2}{|c|}{ Deep } & \multicolumn{2}{|c|}{ Superficial } & \multicolumn{2}{|c|}{ Deep } & \multicolumn{2}{|c|}{ Superficial } \\
\hline & $\boldsymbol{N}$ & $\%$ & $\mathbf{N}$ & $\%$ & $\mathbf{N}$ & $\%$ & $\mathbf{N}$ & $\%$ \\
\hline \multicolumn{9}{|l|}{ Sex } \\
\hline Male $^{a}$ & 275 & 1.2 & 271 & 1.1 & 77 & 1.1 & 107 & 1.5 \\
\hline Female & $485^{b}$ & 1.0 & $833^{d}$ & 1.8 & 166 & 0.9 & $386^{c}$ & 2.1 \\
\hline \multicolumn{9}{|l|}{ Age } \\
\hline $1-44$ years $^{a}$ & 61 & 0.5 & 64 & 0.5 & 3 & 0.5 & 11 & 1.9 \\
\hline $45-64$ years & $140^{\mathrm{b}}$ & 0.8 & $170^{\mathrm{b}}$ & 1.0 & 43 & 0.6 & 82 & 1.2 \\
\hline $65-74$ years & $208^{d}$ & 1.1 & $296^{d}$ & 1.6 & 95 & 0.9 & 179 & 1.8 \\
\hline$\geq 75$ years & $351^{\mathrm{d}}$ & 1.6 & $574^{d}$ & 2.6 & 102 & 1.2 & 221 & 2.6 \\
\hline \multicolumn{9}{|c|}{ Wound contamination } \\
\hline $1+2^{a}$ & 689 & 1.0 & 1044 & 1.5 & 238 & 0.9 & 483 & 1.9 \\
\hline $3+4$ & $68^{\mathrm{d}}$ & 5.1 & $45^{d}$ & 3.3 & $5^{d}$ & 8.9 & $3^{c}$ & 5.4 \\
\hline \multicolumn{9}{|l|}{ ASA score } \\
\hline $1+2^{a}$ & 531 & 0.9 & 772 & 1.4 & 179 & 0.8 & 363 & 1.7 \\
\hline $3+4+5$ & $185^{\mathrm{d}}$ & 2.1 & $287^{d}$ & 3.3 & $51^{c}$ & 1.7 & $104^{\mathrm{d}}$ & 3.5 \\
\hline \multicolumn{9}{|c|}{ Duration of surgery } \\
\hline$\leq P_{75}{ }^{a}$ & 514 & 1.0 & 733 & 1.4 & 167 & 0.8 & 334 & 1.7 \\
\hline$>P_{75}$ & $215^{\mathrm{d}}$ & 1.4 & $334^{d}$ & 2.1 & $76^{b}$ & 1.2 & $159^{d}$ & 2.5 \\
\hline \multicolumn{9}{|c|}{ Emergency surgery } \\
\hline $\mathrm{No}^{\mathrm{a}}$ & 611 & 1 & 902 & 1.5 & 234 & 0.9 & 467 & 1.9 \\
\hline Yes & $148^{\mathrm{d}}$ & 1.6 & $199^{d}$ & 2.2 & $9^{c}$ & 1.1 & $24^{c}$ & 3.0 \\
\hline \multicolumn{9}{|l|}{ Type infection } \\
\hline Superficial ${ }^{a}$ & & & 1104 & 1.6 & & & 493 & 1.9 \\
\hline Deep & 760 & 1.1 & & & 243 & 0.9 & & \\
\hline \multicolumn{9}{|c|}{ NNIS risk index } \\
\hline $0^{\mathrm{a}}$ & 352 & 0.8 & 514 & 1.2 & 124 & 0.8 & 252 & 1.5 \\
\hline 1 & $267^{d}$ & 1.3 & $419^{d}$ & 2.1 & $83^{c}$ & 1.1 & $164^{d}$ & 2.3 \\
\hline 2 & $92^{d}$ & 3.8 & $108^{d}$ & 4.5 & $23^{d}$ & 2.9 & $45^{d}$ & 5.8 \\
\hline 3 & $3^{b}$ & 4.0 & $6^{d}$ & 8.0 & 0 & & 0 & \\
\hline \multicolumn{9}{|c|}{ Pre-operative stay } \\
\hline$\leq 2$ days $^{a}$ & 635 & 1.0 & 943 & 1.4 & 218 & 0.9 & 448 & 1.8 \\
\hline$>2$ days & $125^{\mathrm{d}}$ & 2.6 & $161^{d}$ & 3.3 & $25^{c}$ & 1.9 & $45^{d}$ & 3.4 \\
\hline
\end{tabular}

$N$, number; NNIS, National Nosocomial Infections Surveillance System risk index score; ASA, American Society of Anesthesiologists; $\mathrm{P}_{75}$, 75th percentile; $\mathrm{P}_{75}$ operating time for THA was 100 min.

${ }^{a}$ Reference.

${ }^{b} P<0.05$.

${ }^{c} P<0.01$.

${ }^{d} P<0.001$. 
For THA, a significant association was observed in the univariate analysis between both deep and superficial SSIs and the risk factors: high wound contamination class, high American Society of Anesthesiologists' (ASA) score, duration of surgery longer than the $75^{\text {th }}$ percentile, high National Nosocomial Infections Surveillance System (NNIS) risk index, and pre-operative stay longer than two days. In addition, for THA, a superficial SSI was also associated with: female sex, increasing age and an acute admission.

\section{Multivariate analyses}

In the multivariate analyses (Table III), the NNIS risk index, composed of ASA score, wound contamination class and duration of surgery, was used instead of using these factors separately because of the low numbers of patients with high ASA scores and wound contamination classes. In univariate analyses, the $P$ values for sex, age, NNIS risk index, emergency surgery and pre-operative stay were $<0.20$, both for all procedures together and for THA separately, and were included in the multivariate models. 
Table III.

Odds ratios (OR) with $95 \%$ confidence intervals (95\% CI) for factors associated with the development of a deep or superficial surgical site infection (SSI) for all procedures and total hip arthroplasty alone, mean of the individual predicted probabilities of developing an SSI calculated by the model for patients with and without an SSI, and their ratio by multivariate analysis

\begin{tabular}{|c|c|c|c|c|c|c|c|c|}
\hline & \multicolumn{4}{|c|}{ All procedures $(N=64598)$} & \multicolumn{4}{|c|}{ THA $(N=24307)$} \\
\hline & \multicolumn{2}{|c|}{ Deep SSI } & \multicolumn{2}{|c|}{$\begin{array}{c}\text { Superficial } \\
\text { SSI }\end{array}$} & \multicolumn{2}{|c|}{ Deep SSI } & \multicolumn{2}{|c|}{$\begin{array}{c}\text { Superficial } \\
\text { SSI }\end{array}$} \\
\hline & $\begin{array}{l}\text { Adj } \\
\text { OR }\end{array}$ & $\begin{array}{c}95 \% \\
\text { CI }\end{array}$ & $\begin{array}{l}\text { Adj } \\
\text { OR }\end{array}$ & $\begin{array}{l}95 \% \\
\text { CI }\end{array}$ & $\begin{array}{l}\text { Adj } \\
\text { OR }\end{array}$ & $\begin{array}{c}95 \% \\
\text { CI }\end{array}$ & $\begin{array}{l}\text { Adj } \\
\text { OR }\end{array}$ & $\begin{array}{c}95 \% \\
\text { CI }\end{array}$ \\
\hline \multicolumn{9}{|l|}{ Sex } \\
\hline Male & $1.0^{\mathrm{a}}$ & & $1.0^{\mathrm{a}}$ & & & & $1.0^{\mathrm{a}}$ & \\
\hline Female & 0.7 & $\begin{array}{l}0.6- \\
0.9\end{array}$ & 1.2 & $\begin{array}{l}1.1- \\
1.4\end{array}$ & & & 1.3 & $\begin{array}{l}1.1- \\
1.7\end{array}$ \\
\hline \multicolumn{9}{|l|}{ Age } \\
\hline $1-44$ years & $1.0^{\mathrm{a}}$ & & $1.0^{\mathrm{a}}$ & & $1.0^{\mathrm{a}}$ & & $1.0^{\mathrm{a}}$ & \\
\hline $45-64$ years & 1.3 & $\begin{array}{l}1.0- \\
1.8\end{array}$ & 1.4 & $1-1.8$ & 1.5 & $\begin{array}{l}0.4- \\
4.9\end{array}$ & 0.6 & $\begin{array}{l}0.3- \\
1.1\end{array}$ \\
\hline $65-74$ years & 1.7 & $\begin{array}{l}1.3- \\
2.4\end{array}$ & 1.9 & $\begin{array}{l}1.4- \\
2.5\end{array}$ & 2.1 & $\begin{array}{l}0.6- \\
6.7\end{array}$ & 0.8 & $\begin{array}{l}0.4- \\
1.6\end{array}$ \\
\hline$\geq 75$ years & 2.3 & $\begin{array}{l}1.7- \\
3.1 \\
\end{array}$ & 2.8 & $\begin{array}{l}2.1- \\
3.8\end{array}$ & 2.5 & $\begin{array}{l}0.8- \\
8.0\end{array}$ & 1.1 & $\begin{array}{l}0.6- \\
2.1\end{array}$ \\
\hline \multicolumn{9}{|l|}{ Emergency surgery } \\
\hline No & $1.0^{\mathrm{a}}$ & & $1.0^{\mathrm{a}}$ & & & & $1.0^{\mathrm{a}}$ & \\
\hline Yes & 1.4 & $\begin{array}{l}1.1- \\
1.7 \\
\end{array}$ & 1.2 & $\begin{array}{l}1.0- \\
1.4 \\
\end{array}$ & & & 1.4 & $\begin{array}{l}0.9- \\
2.3 \\
\end{array}$ \\
\hline \multicolumn{9}{|l|}{ NNIS risk index } \\
\hline 0 & $1.0^{\mathrm{a}}$ & & $1.0^{\mathrm{a}}$ & & $1.0^{\mathrm{a}}$ & & $1.0^{\mathrm{a}}$ & \\
\hline 1 & 1.4 & $\begin{array}{l}1.2- \\
1.7\end{array}$ & 1.4 & $\begin{array}{l}1.2- \\
1.6\end{array}$ & 1.4 & $\begin{array}{l}1.0- \\
1.9\end{array}$ & 1.6 & $\begin{array}{l}1.3- \\
2.0\end{array}$ \\
\hline 2 & 3.3 & $\begin{array}{l}2.5- \\
4 .\end{array}$ & 2.5 & $2-3.1$ & 3.1 & $\begin{array}{l}1.9- \\
5.1\end{array}$ & 3.6 & $\begin{array}{l}2.5- \\
5.2\end{array}$ \\
\hline 3 & 2.7 & $\begin{array}{l}0.8- \\
8.8\end{array}$ & 4.7 & $\begin{array}{l}1.9- \\
11.4 \\
\end{array}$ & - & & & - \\
\hline \multicolumn{9}{|l|}{ Pre-operative stay } \\
\hline$\leq 2$ days & $1.0^{\mathrm{a}}$ & & $1.0^{\mathrm{a}}$ & & $1.0^{\mathrm{a}}$ & & $1.0^{\mathrm{a}}$ & \\
\hline$>2$ days & 2.0 & $\begin{array}{l}1.6- \\
2.4\end{array}$ & 1.7 & $1.4-2$ & 1.9 & $\begin{array}{l}1.2- \\
3.0 \\
\end{array}$ & 1.6 & $\begin{array}{l}1.1- \\
2.3\end{array}$ \\
\hline Hospital & $0.4^{c}$ & $\begin{array}{l}0.2- \\
0.6\end{array}$ & $0.6^{c}$ & $\begin{array}{l}0.3- \\
0.8\end{array}$ & $0.5^{\mathrm{b}}$ & $\begin{array}{l}0.2- \\
0.8\end{array}$ & $0.7^{c}$ & $\begin{array}{l}0.3- \\
1.0\end{array}$ \\
\hline $\begin{array}{l}\text { Mean of predicted probability } \\
\text { with the model for patients } \\
\text { with an SSI }\end{array}$ & 0.021 & & 0.037 & & 0.018 & & 0.041 & \\
\hline $\begin{array}{l}\text { Mean of predicted probability } \\
\text { with the model for patients } \\
\text { without an SSI }\end{array}$ & 0.011 & & 0.016 & & 0.009 & & 0.018 & \\
\hline Ratio of means & 1.9 & & 2.3 & & 1.9 & & 2.3 & \\
\hline
\end{tabular}

Adj OR, adjusted odds ratio; NNIS, National Nosocomial Infections Surveillance System risk index score.

${ }^{a}$ Reference.

b $P<0.01$.

c $P<0.001$. 
For THA, a significant association was found in the multivariate analysis between deep SSI and the following factors: NNIS risk index and pre-operative stay longer than two days. A significant association was also found between a superficial SSI and the factors: female sex, NNIS risk index, and pre-operative stay longer than two days.

The predictive power of the multivariate models was low. For the group with a real SSI, the mean predicted probability of getting an SSI with the model ranged from 0.009 to 0.041 . These values were slightly higher than those for the patients without an SSI. The ratio of the means ranged from 1.9 to 2.3. This indicates that the predicted probability of developing an SSI with the model was 1.9 to 2.3 times higher for patients with SSIs compared with patients without SSIs (Table III). Estimates of risk factors calculated with a logistic regression model were further from the null than the estimates acquired with the random effect model (data not shown). Moreover, the confidence limits of the estimates with the logistic regression model were smaller.

\section{Discussion}

This study underlines the usefulness of a random effect model, which adjusts risk estimates for random variation between hospitals, in a multicentre study on risk factors for SSIs. It was also useful to gain insight into the distribution of the most widespread bacterial species responsible for SSIs. However, this study has two limitations. First, other hospital-related factors may have influenced the measure of the clustering of bacteria. Second, the study did not explore the additional use of random effect models in which regression coefficients also differed between hospitals.

A decreasing trend was seen in the incidence of superficial SSIs after THA. This trend may be interpreted as an effect of surveillance since most hospitals in our analyses have performed surveillance for SSI after THA for many years. This trend is in agreement with earlier results from our surveillance network. ${ }^{10}$

Bacteriological documentation was missing for $33 \%$ of the superficial SSIs and $32 \%$ of the deep SSIs. According to the infection control professionals, the diagnosis of an SSI by a physician on the basis of clinical symptoms alone was the most frequent reason for not taking a specimen for culture. The lack of laboratory confirmation is unlikely to have introduced bias, but may have caused a decrease in precision. However, it is advisable to take a culture in all cases to gain good insight into the incidence of nosocomial infections and to detect small outbreaks of pathogens. Clustering of bacteria species was an important phenomenon in SSI. Continuous surveillance of bacteria, including detection of clusters within hospitals, could help to direct infection control practice in preventing cross-transmission.

The species found in this study were in accordance with other studies, but the proportions differed. ${ }^{15}$ and 16 Staphylococci accounted for most infections in the Dutch hospitals, and only a few deep SSIs (1.5\%) were caused by anaerobes.

As in other countries, $S$. aureus and CNS were the most frequent pathogens associated with SSIs in The Netherlands. ${ }^{2}$ and 16 S. aureus was the dominant pathogen and its contribution seemed to be increasing. Although the resistance of bacteria is not recorded in the PREZIES database, it is not probable that methicillin-resistant $S$. aureus (MRSA) caused this increase as it is known from other surveillance data that the prevalence of MRSA in The Netherlands has remained very low. ${ }^{17}$ In contrast to $S$. aureus, the incidence of CNS seemed to 
decrease. A possible explanation for the observed decrease of CNS over time may be the changing opinion about the importance of CNS in superficial samples. In 1996, CNS in a sample was believed to be the cause of an SSI. However, in later years, microbiologists realized that CNS are a major part of human skin flora and that CNS in a sample from a superficial SSI could be a contaminant rather than the source of the infection. This change of view could have influenced the registration of CNS. Nevertheless, this decreasing trend was also seen for deep SSIs. No change in view occurred for deep SSIs, so the decrease is real. ${ }^{18}$ This decrease in CNS as a pathogen responsible for SSI is in contrast with previous work. ${ }^{19}$ and 20

Logistic regression models ignore interhospital variability, such as the small outbreaks of pathogens observed in this study. Thus, logistic regression would overestimate the contribution of patient-related factors, as confirmed in the present data. Therefore, a random effect model was used in this study, which took account of hospital-related variability in the risk of developing an SSI after orthopaedic procedures.

Most patient-related risk factors had a low predictive power for the development of an SSI. The data show only small differences in infection rates between age groups, NNIS scores and ASA scores (Table II). As a result, the predicted probability for developing an SSI in the multivariate models was low for both superficial and deep SSIs. This is in line with earlier results from the same dataset. ${ }^{10} \mathrm{~A}$ pre-operative stay longer than two days was the only factor to be significantly associated with the risk of an SSI in most models. The CDC guidelines state that pre-operative stay is likely to be a marker for other factors associated with SSIs, such as severity of illness and comorbidity. ${ }^{1}$

The predictive power of the multivariate models based on the patient-related risk factors registered in our surveillance data is low. For the prevention of SSIs, it is important to gain a clear understanding of the major factors associated with the risk of SSI development. These could include patient-related factors such as diabetes or an altered immune response, and also hospital-related factors. Factors such as the presence of a $S$. aureus carrier in a hospital or specific hygienic deficiencies may make an important contribution to the variation in SSI rates between hospitals. Detailed information about the antibiotic prophylaxis regimes in hospitals is also likely to be relevant. Therefore, consideration should be given to which patient-related, operation-room-related and hospital-related factors to include in SSI surveillance. Analysis with random effect models is useful to account for interhospital variation in nosocomial surveillance systems.

\section{Acknowledgements}

The authors thank N. Nagelkerke for statistical advice and $\mathrm{H}$. de Neeling for sharing microbiology knowledge. They also thank F. Heathcoth, $\mathrm{H}$. de Ronde and the members of the PREZIES team, in particular T. Lau, J. Mannien and T. van der Kooi for helpful discussions.

\section{References}

1 Centers for Disease Control and Prevention, Healthcare Infection Control Practices Advisory Committee, Guideline for prevention of surgical site infection, 1999, Am J Infect Control 27 (1999), pp. $97-132$. 
2 J. Segreti, Prosthetic joint infections, Curr Treat Opt Infect Dis 2 (2000), pp. 200-207.

3 E.F. Berbari, A.D. Hanssen and M.C. Duffy et al., Risk factors for prosthetic joint infection: casecontrol study, Clin Infect Dis 27 (1998), pp. 1247-1254.

4 Dutch Working Party on Infection Prevention, Infection prevention in operating rooms, Dutch Working Party on Infection Prevention, Leiden (2003) p. 1-16.

5 Dutch Working Party on Infection Prevention, Registration of nosocomial infections, Dutch Working Party on Infection Prevention, Leiden (2003) p. 1-14.

6 F. Lecuire, D. Gontier and J. Carrere et al., Ten-year surveillance of nosocomial surgical site infections in an orthopaedic surgery department [in French], Rev Chir Orthop Reparatrice Appar Mot 89 (2003), pp. 479-486.

7 J. Kluytmans, H. Berg and P. Steegh et al., Outbreak of Staphylococcus schleiferi wound infections: strain characterization by randomly amplified polymorphic DNA analysis, PCR ribotyping, conventional ribotyping and pulsed-field gel electrophoresis, J Clin Microbiol 36 (1998), pp. 2214-2219.

8 R.J. Jarvis and S. Zaza, Investigation of outbreaks. In: C.G. Mayhall, Editor, Hospital epidemiology and infection control (2nd ed), Williams and Wilkins, Philadelphia (1999), pp. 111-120.

9 A.R. Localio, J.A. Berlin and T.R. Ten Have et al., Adjustments for center in multicenter studies: an overview, Ann Intern Med 135 (2001), pp. 112-123.

10 Geubbels ELPE. Prevention of surgical site infections through surveillance. Thesis. Utrecht; 2002. p. $1-151$.

11 PREZIES. Prevention of nosocomial infections by surveillance [in Dutch]. Bilthoven: RIVM/CBO; 2003.

12 G. Walenkamp, Surveillance of surgical-site infections in orthopedics, Acta Orthop Scand 74 (2003), pp. 172-174.

13 Dutch Institute for Healthcare, Revision consensus total hip prostheses [in Dutch], CBO, Utrecht (1994).

14 T.A.B. Snijders and R.J. Bosker, Multilevel analysis: an introduction to basic and advanced multilevel modeling, Sage, London (1999).

15 A.F. Widmer, New developments in diagnosis and treatment of infection in orthopaedic implants, Clin Infect Dis 33 (2001) (Suppl 2), pp. 94-106.

16 D.P. Lew, D. Pittet and F.A. Waldvogel, Infections that complicate the insertion of prosthetic devices. In: C.G. Mayhall, Editor, Hospital epidemiology and infection control (2nd ed), Williams and Wilkins, Philadelphia (1999), pp. 937-957.

17 SWAB. NETHMAP, Consumption of antimicrobial agents and antimicrobial resistance among medically important bacteria in The Netherlands, SWAB/RIVM, Rotterdam (2004).

18 H.A. Verbrugh, R.P. Mouton and A.M. Polderman, Medical microbiology [in Dutch] (8th ed), Bohn Stafleu Van Loghum, Houten/Zaventem (1992).

19 S.A.J. Zaat, J. Boelens and J. Dankert, New insights in the pathogeneses of biomaterial-related infections [in Dutch], Ned Tijd Med Microbiol 10 (2002), pp. 17-24.

20 G.H. Walenkamp, Prevention and treatment of infection in orthopaedic surgery and traumatology, Surg Tech Orthop Traumatol 55-010-C-10 (2000), pp. 1-11. 